\title{
A multiplex-system to target 16 male-specific and 15 autosomal genetic markers for orang-utans (genus: Pongo)
}

Nietlisbach, Pirmin ; Nater, Alexander ; Greminger, Maja P ; Arora, Natasha ; Krützen, Michael

DOI: https://doi.org/10.1007/s12686-010-9278-2

Posted at the Zurich Open Repository and Archive, University of Zurich ZORA URL: https://doi.org/10.5167/uzh-71190

Journal Article

Published Version

Originally published at:

Nietlisbach, Pirmin; Nater, Alexander; Greminger, Maja P; Arora, Natasha; Krützen, Michael (2010). A multiplex-system to target 16 male-specific and 15 autosomal genetic markers for orang-utans (genus: Pongo). Conservation Genetics Resources, 2(S1):153-158.

DOI: https://doi.org/10.1007/s12686-010-9278-2 


\title{
A multiplex-system to target $\mathbf{1 6}$ male-specific and $\mathbf{1 5}$ autosomal genetic markers for orang-utans (genus: Pongo)
}

\author{
Pirmin Nietlisbach • Alexander Nater • \\ Maja P. Greminger · Natasha Arora • \\ Michael Krützen
}

Received: 30 June 2010/Accepted: 6 July 2010/Published online: 25 July 2010

(C) Springer Science+Business Media B.V. 2010

\begin{abstract}
Genetic studies of dispersal on local spatial and short temporal scales require a large number of autosomal microsatellites. However, the study of dispersal over large spatial scales and the resolution of deep evolutionary histories require marker systems that are preferentially inherited through the male or female line. Addressing such questions in endangered orang-utans (genus: Pongo) bears significant relevance to species conservation, as habitat destruction and fragmentation pose a significant threat to the whole genus. Here, we report 16 male-specific markers (nine human-derived microsatellites, six single nucleotide and one insertion-deletion polymorphisms), and 15 novel Pongo-derived autosomal microsatellite loci. All 31 markers can be amplified in four multiplex polymerase chain reactions even in DNA derived from faecal material. The markers can be applied to studying a wide range of important questions in this genus, such as conservation genetics, social structure, phylogeny and phylogeography.
\end{abstract}

Keywords Pongo spp.

Single nucleotide polymorphisms · Microsatellites . Y chromosome $\cdot$ SNP typing $\cdot$ Non-invasive samples

Electronic supplementary material The online version of this article (doi:10.1007/s12686-010-9278-2) contains supplementary material, which is available to authorized users.

P. Nietlisbach - A. Nater · M. P. Greminger · N. Arora ·

M. Krützen ( $\square)$

Evolutionary Genetics Group, Anthropological Institute

and Museum, University of Zurich, Winterthurerstrasse 190,

8057 Zurich, Switzerland

e-mail: michael.kruetzen@aim.uzh.ch
The endangered orang-utans occur on the islands of Borneo (Pongo pygmaeus; about 50,000 animals) and Sumatra (P. abelii; about 6,500 animals), where they have undergone a recent dramatic decline in population size (Goossens et al. 2006; Wich et al. 2008). This has been mostly attributed to habitat loss, leading to heavily fragmented populations of often only a few hundred individuals (Wich et al. 2008). Therefore, it is essential to maintain genetic diversity, which has been linked to population fitness (e.g. Reed and Frankham 2003). This can be achieved by maintaining corridors between fragmented populations, allowing animals to follow natural dispersal patterns (Gilbert-Norton et al. 2010).

Studying natural dispersal in wild orang-utans pose significant challenges. Behavioural observations suggested higher male than female dispersal (Delgado and van Schaik 2000), although this has not been fully confirmed by previous genetic studies (Utami et al. 2002; Goossens et al. 2005), where patterns of direct dispersal were investigated using autosomal microsatellite markers. However, direct inferences from autosomal markers are limited to the timescale of a few generations and geographically small areas, as sexual recombination will break down sex-specific information (Goudet et al. 2002). Sex-biased dispersal over larger time and spatial scales can be investigated by contrasting genetic information obtained from markers inherited through either the male or female lineage (Handley and Perrin 2007).

In orang-utans, maternally transmitted mitochondrial DNA markers are widely available (e.g. Warren et al. 2001), but markers on the male-specific region of the $\mathrm{Y}$ chromosome have not yet been applied. Here, we report 16 malespecific markers for the application in the genus Pongo. Nine of these markers are human-derived microsatellite loci, six are single nucleotide polymorphisms (SNPs) and one is an 
insertion-deletion polymorphism (indel). We also provide 15 novel orang-utan specific autosomal microsatellite markers, which yielded more reliable amplification results for use with highly degraded faecal samples than humanderived markers (data not shown).

In a previous study (Erler et al. 2004), 45 male-specific human-derived loci were shown to amplify in orangutans. Of those, we selected 25 loci re-sequenced them, designed orang-utan specific primers, and tested for levels of polymorphism in 14 Bornean and Sumatran orangutans (Table 1; see laboratory procedures below). After aligning flanking regions of all 25 microsatellite loci, we discovered two SNPs and one indel. Additionally, we identified four more SNPs by sequencing $\mathrm{Y}$ chromosome conserved anchored tagged sequence loci DBY13, SMCY12, and SMCY14 (Hellborg and Ellegren 2003). We combined 16 Y-specific polymorphic markers (GenBank Acc.No. HM803995-HM804006) into two multiplex PCRs (Table 1). Actual repeat number was linked to amplicon length through sequence data (Table S1 in the Online Resources). For SNP typing, we applied a modified fragment length discrepant allele specific PCR ( $\mathrm{Li}$ et al. 2009). Each PCR combined a fluorescently labelled forward primer with two interrogating reverse primers. The reverse primers were complementary to one of each SNP allele at their $3^{\prime}$-end and differed in their length at the $5^{\prime}$-end; the resulting difference in product length allowed for allelic discrimination by fragment length analysis (Fig. 1a). One microsatellite locus had a SNP in close proximity and another a SNP and an indel, which allowed for simultaneous screening in one or two PCRs, respectively (Fig. 1). Multiplex reactions for $\mathrm{Y}$ markers consisted of $1 \mu \mathrm{l}$ DNA template in an $8 \mu \mathrm{l}$ volume, and were carried out with the Qiagen PCR Multiplex Kit according to manufacturer's instructions and varying primer concentrations (Table 1). In addition to the manufacturer's recommendations, we also included a touchdown step in the PCRs, starting at $4^{\circ} \mathrm{C}$ above the reported annealing temperature in Table 1 , then decreasing by $0.5^{\circ} \mathrm{C}$ per cycle for eight cycles to reach the annealing temperature for the last 40 cycles. We assessed the variability of the Y-markers on a population level in 21 and 14 orang-utans from one Bornean (subspecies P. pygmaeus wurmbii) and one Sumatran site, respectively. In addition, we report the number of Y-polymorphisms in an extensive sample of 173 animals covering most of the range of the genus Pongo (Table 1, Nietlisbach 2009). We tested scoring reliability by repeating $32 \%$ of all genotyping reactions, and found an average scoring error rate of $0.6 \%$ over all loci. It has not escaped our notice that our results immediately suggest a possible diagnostic system for elucidating species affiliation or hybrid status for males of this genus
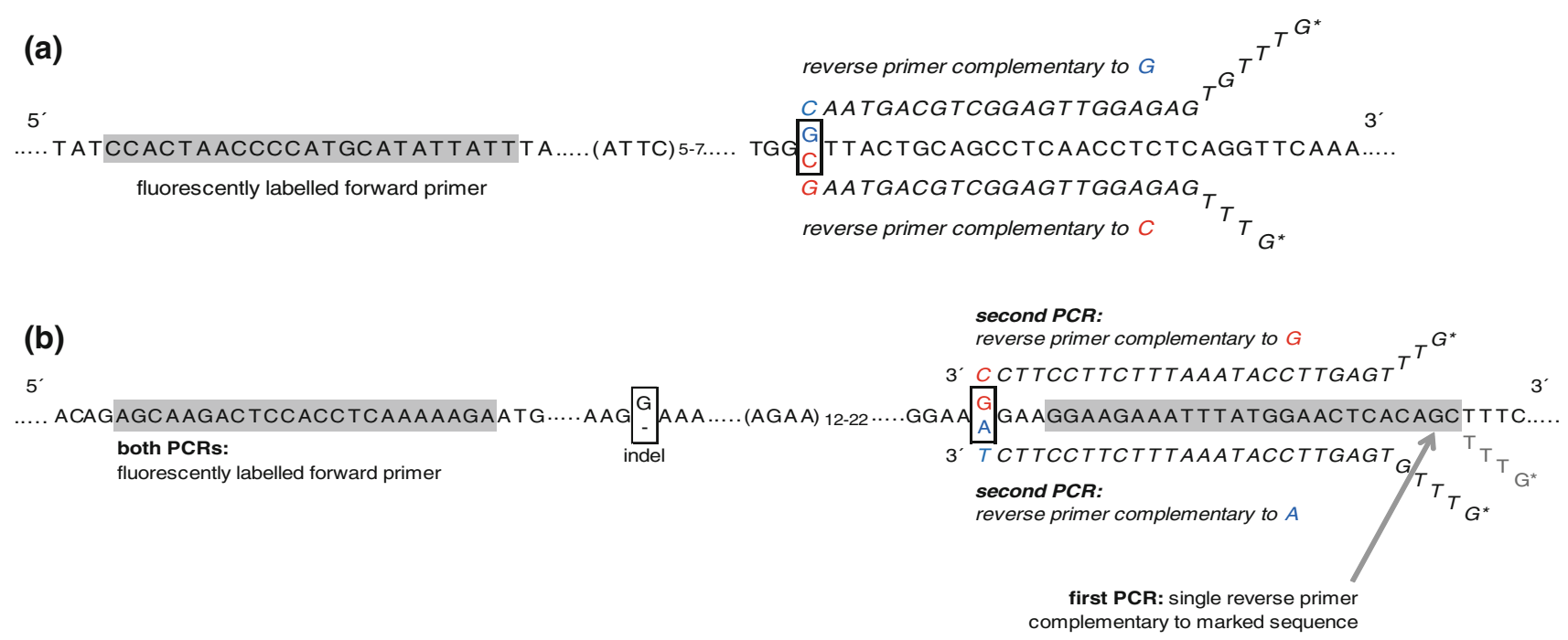

Fig. 1 Schematic representation of genotyping techniques for combined microsatellite/SNP typing. a Genotyping of SNP and microsatellite locus (DYS577). This locus was typed by a modified fragment length discrepant allele specific PCR (see text, Li et al. 2009) which targets the SNP in the box and the repeat motif of the microsatellite in one PCR. Discrimination between the two states of the SNP was achieved through competition of two reverse primers differing in length; and both reverse primers having a 1 to $2^{\circ} \mathrm{C}$ lower melting temperature and higher concentration than the fluorescently labelled forward primer (Table 1) to increase competition. Calibration was done with samples of known sequence. $\mathbf{b}$ Typing of three markers in two PCRs (DYS630). A first PCR using the grey primers amplified the region containing all three markers. A second PCR discriminates between SNP states as described in Fig. 1a. Due to confounding effects of the polymorphic microsatellite and indel, amplicon sizes of the first and second reactions have to be compared. If the amplicon of the first reaction is 5 bases longer than that of the second reaction, the SNP allele at this locus is G; if the difference is 3 bases, the SNP state is A. * PIG-tails were added to the $5^{\prime}$-end of reverse primers to enhance adenylation (Brownstein et al. 1996) 


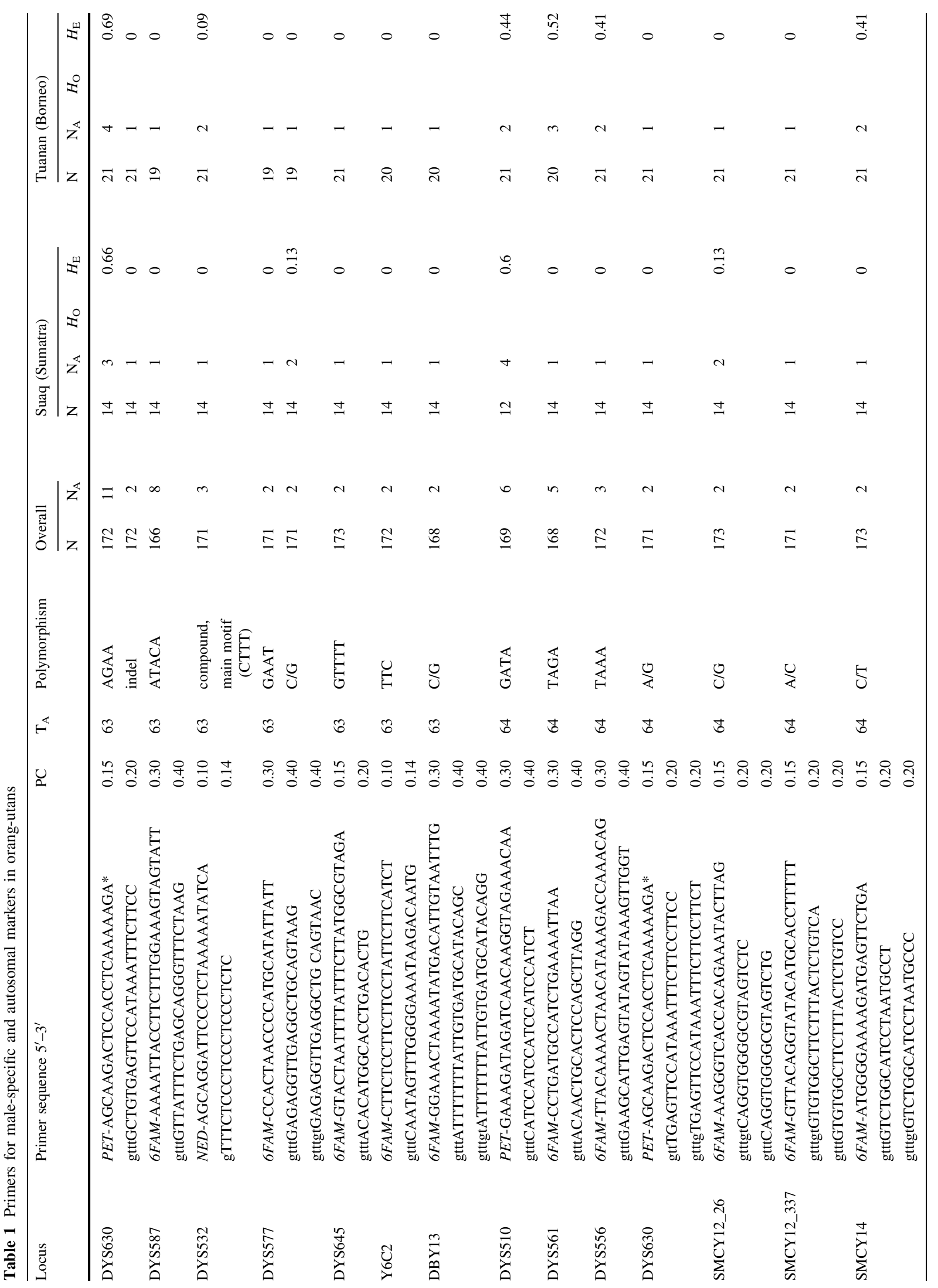




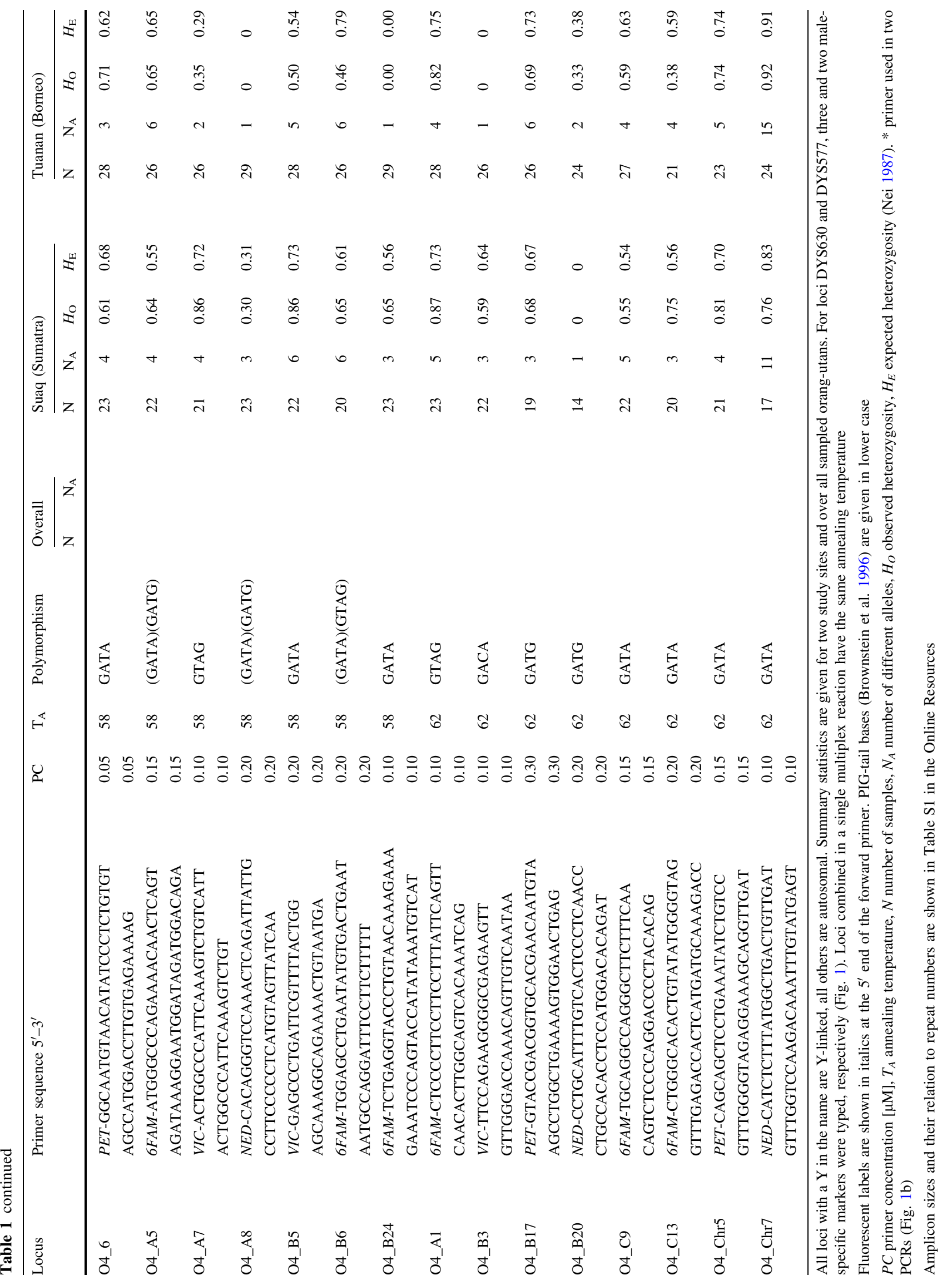


(Nietlisbach 2009), if used in unison with readily available mtDNA markers.

To clone autosomal microsatellite markers, we extracted genomic DNA from $25 \mathrm{mg}$ of frozen muscle tissue from a Sumatran orang-utan, using the DNeasy Tissue Kit (Qiagen). We digested ten micrograms of the purified DNA with Nhe I and AluI (New England Biolabs) and sizeselected for fragments between 400 and 1,200 base pairs length. Enrichment, cloning and sequencing were carried out as described in Nater et al. (2008), using only tetranucleotide biotinylated probes $\left[(\mathrm{GACA})_{7},(\mathrm{GATA})_{7}\right.$, and $\left.(\mathrm{GATC})_{7}\right]$. We sequenced plasmids from 68 positive clones, of which $70 \%$ contained a microsatellite repeat. For 25 loci, which contained long uninterrupted repeats, we designed primers and amplified these loci in twelve orangutans. Levels of polymorphism were qualitatively assessed on high-resolution Spreadex gels (Elchrom Scientific). Based on these results, we fluorescently labelled the forward primers of the 15 most polymorphic markers and combined these 15 loci (GenBank Acc.No. HM804007HM804021) into two multiplex PCRs (Table 1). Then, we genotyped 29 orang-utans from Borneo and 23 from Sumatra, using DNA extracts from faecal samples with target DNA concentration ranging from 25 to $1,000 \mathrm{pg} / \mu \mathrm{l}$, strictly following guidelines from Morin et al. (2001). PCRs using the Qiagen PCR Multiplex Kit contained $1 \mu \mathrm{l}$ template DNA in an $8 \mu \mathrm{l}$ final volume, with varying primer concentrations and annealing temperatures (Table 1). PCRs included 45 cycles with conditions according to manufacturer's instructions.

If not indicated otherwise, we used standard laboratory techniques at each step. We designed PCR primers with the PrimerSelect software implemented in Lasergene v7 (DNASTAR). PCR amplifications were performed on Veriti 96-well thermal cyclers (Applied Biosystems). Sequencing reactions were carried out using the BigDye Terminator v3.1 on a 3730 DNA Analyzer (both Applied Biosystems) according to manufacturer's instructions, cleaned-up using a $\mathrm{MgSO}_{4}$ precipitation procedure, fol-

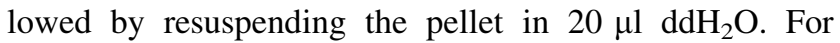
fragment length analysis, PCR products were diluted 20-80 times in $\mathrm{ddH}_{2} \mathrm{O}$. One microlitre of this was added to $9.93 \mu \mathrm{l}$ HiDi formamide and $0.07 \mu 1$ of GeneScan 500 LIZ Size Standard (both Applied Biosystems) and denatured for three minutes at $95^{\circ} \mathrm{C}$. We ran the samples on a 3730 DNA Analyzer and obtained genotypes using GeneMapper software v4.0 (Applied Biosystems). For the statistical analyses, we used MStools v3.1 add-into Microsoft Excel (Park 2001) and Genepop v4.0 (Rousset 2008).

Fragment length discrepant allele specific PCR used as SNP typing technique proved to be a reliable and costefficient strategy to assess SNP variation. The possibility to combine this technique with conventional microsatellite fragment length analysis makes it a suitable method to include a small number of SNPs to complement an extensive microsatellite analysis. The polymorphic male-specific markers for orang-utans described here promise to be highly useful for population genetic and phylogenetic studies addressing questions about dispersal strategies, phylogeographic patterns, and comparisons with other molecular markers. The autosomal markers can be applied to investigate local dispersal or assess relatedness and paternity. Knowledge about such processes, in particular about natural dispersal strategies, is important for species conservation.

Acknowledgments We thank C. van Schaik, M. van Noordwijk, J. Pamungkas, and D. Perwitasari-Farajallah. We are also indebted to all individuals who helped collecting samples in the field. This study was funded by the Swiss National Science Foundation (31003A116848 to MK), Messerli Foundation, A.H.-Schultz Stiftung, and Claraz Schenkung. We thank the Indonesian Institute of Sciences (LIPI), the Indonesian State Ministry for Research and Technology (RISTEK), and the Sabah Wildlife Department for granting permission to undertake this research. All sampling and transportation of samples was conducted in accordance with Indonesian, Malaysian and international regulations (CITES).

\section{References}

Brownstein MJ, Carpten JD, Smith JR (1996) Modulation of nontemplated nucleotide addition by Taq DNA polymerase: primer modifications that facilitate genotyping. Biotechniques 20(6): 1004-1010

Delgado RA, van Schaik CP (2000) The behavioral ecology and conservation of the orang-utan (Pongo pygmaeus): a tale of two islands. Evol Anthropol 9(5):201-218

Erler A, Stoneking M, Kayser M (2004) Development of Y-chromosomal microsatellite markers for nonhuman primates. Mol Ecol 13(10):2921-2930. doi:10.1111/j.1365-294X.2004. 02304.x

Gilbert-Norton L, Wilson R, Stevens JR, Beard KH (2010) A metaanalytic review of corridor effectiveness. Conserv Biol 24(3): 660-668. doi:10.1111/j.1523-1739.2010.01450.x

Goossens B, Chikhi L, Jalil MF, Ancrenaz M, Lackman-Ancrenaz I, Mohamed M, Andau P, Bruford MW (2005) Patterns of genetic diversity and migration in increasingly fragmented and declining orang-utan (Pongo pygmaeus) populations from Sabah, Malaysia. Mol Ecol 14(2):441-456. doi:10.1111/j.1365-294X.02421.x

Goossens B, Chikhi L, Ancrenaz M, Lackman-Ancrenaz I, Andau P, Bruford MW (2006) Genetic signature of anthropogenic population collapse in orang-utans. PLoS Biol 4(2):285-291. doi: e2510.1371/journal.pbio.0040025

Goudet J, Perrin N, Waser P (2002) Tests for sex-biased dispersal using bi-parentally inherited genetic markers. Mol Ecol 11(6):1103-1114

Handley LJL, Perrin N (2007) Advances in our understanding of mammalian sex-biased dispersal. Mol Ecol 16(8):1559-1578. doi:10.1111/j.1365-294X.2006.03152.x

Hellborg L, Ellegren H (2003) Y chromosome conserved anchored tagged sequences (YCATS) for the analysis of mammalian malespecific DNA. Mol Ecol 12(1):283-291

Li SZ, Wan HR, Ji HY, Zhou KY, Yang G (2009) SNP discovery based on CATS and genotyping in the finless porpoise 
(Neophocaena phocaenoides). Conserv Genet 10(6):2013-2019. doi:10.1007/s10592-009-9882-4

Morin PA, Chambers KE, Boesch C, Vigilant L (2001) Quantitative polymerase chain reaction analysis of DNA from noninvasive samples for accurate microsatellite genotyping of wild chimpanzees (Pan troglodytes verus). Mol Ecol 10(7):1835-1844

Nater A, Krützen M, Lindholm AK (2008) Development of polymorphic microsatellite markers for the livebearing fish Poecilia parac. Mol Ecol Resour 8(4):857-860. doi:10.1111/ j.1755-0998.2008.02090.x

Nei M (1987) Molecular evolutionary genetics. Columbia University Press, New York

Nietlisbach P (2009) Male-specific markers in orang-utans (Pongo spp.)-Dispersal and phylogeny. MSc thesis. University of Zurich

Park SDE (2001) Trypanotolerance in West African cattle and the population genetic effects of selection. University of Dublin, Dublin, Ireland
Reed DH, Frankham R (2003) Correlation between fitness and genetic diversity. Conserv Biol 17(1):230-237

Rousset F (2008) GENEPOP '007: a complete re-implementation of the GENEPOP software for Windows and Linux. Mol Ecol Resour 8(1):103-106. doi:10.1111/j.1471-8286.2007.01931.x

Utami SS, Goossens B, Bruford MW, de Ruiter JR, van Hooff J (2002) Male bimaturism and reproductive success in Sumatran orang-utans. Behav Ecol 13(5):643-652

Warren KS, Verschoor EJ, Langenhuijzen S, Heriyanto, Swan RA, Vigilant L, Heeney JL (2001) Speciation and intrasubspecific variation of Bornean orang-utans, Pongo pygmaeus pygmaeus. Mol Biol Evol 18 (4):472-480

Wich SA, Meijaard E, Marshall AJ, Husson S, Ancrenaz M, Lacy RC, van Schaik CP, Sugardjito J, Simorangkir T, Traylor-Holzer K, Doughty M, Supriatna J, Dennis R, Gumal M, Knott CD, Singleton I (2008) Distribution and conservation status of the orang-utan (Pongo spp.) on Borneo and Sumatra: how many remain? Oryx 42(3):329-339. doi:10.1017/s003060530800197x 